\title{
The Benefits of High Intensity Exercise on the Brain of a Drug Abuser
}

\author{
Daniel A. R. Cabral ${ }^{1}$, Vagner D. O. Tavares ${ }^{1}$, Kell G. da Costa $^{1}$, Paulo H. D. Nascimento ${ }^{1}$, Heloiana K. C. Faro ${ }^{1}$, \\ Hassan M. Elsangedy ${ }^{1} \&$ Eduardo B. Fontes ${ }^{1}$ \\ ${ }^{1}$ NEUROex: Research Group in Physical Activity, Cognition and Behavior, Health Science Center, Department of \\ Physical Education, Federal University of Rio Grande do Norte, Natal/RN, Brazil \\ Correspondence: Eduardo Bodnariuc Fontes, Federal Universiry of Rio Grande do Norte, Natal, RN, Brazil. Tel: \\ 55-849-898-9800. E-mail: eduardobfontes@gmail.com
}

\author{
Received: March 31, 2018 Accepted: April 25, 2018 Online Published: May 9, 2018 \\ doi:10.5539/gjhs.v10n6p123 \\ URL: https://doi.org/10.5539/gjhs.v10n6p123
}

\begin{abstract}
Chronic drug abuse has been shown to cause dysfunctions on the frontal lobe and affect cognition, cardiac autonomic control and psychosocial aspects. Despite physical exercise has been shown to improve cerebral functioning, the effects of a high intensity exercise training program needs to be further explored in a drug abuse condition. The patient was a 32-year-old male who has been an alcohol and crack/cocaine user for 20 years. The high intensity exercise training protocol consisted of four 30-second "all-out" bouts performed three times per week during four weeks. The participant had electroencephalographic (EEG) activity, cognition, cardiac autonomic control and psychosocial questionnaires evaluated before and after high intensity exercise training. Prefrontal cortex (PFC) oxygenation during an incremental running exercise test was also recorded. EEG topographical analysis revealed greater PFC activation during the cognitive test. Performance on the cognitive test was enhanced (1 number of total errors and reaction time). Parasympathetic cardiac indices, including RMSSD, SDNN, Pnn50\% and HF power increased by $77.4 \%, 83.3 \%, 57.7 \%$ and $293.2 \%$, respectively. Sleep quality increased $23 \%$ and anxiety levels decreased $52.6 \%$. Psychological and social domains increased $5.3 \%$ and $13.7 \%$, respectively. In addition, incremental treadmill running time increased $12.5 \%$ and PFC oxyhemoglobin increased $228.2 \%$ at the beginning of the treadmill test, $305.4 \%$ at the middle and $359.4 \%$ at the end of the test. Thus, high intensity exercise training improved PFC functioning, cardiac autonomic control and psychological parameters. These results might indicate high intensity exercise as an alternative and non-pharmacological tool to help the rehabilitation of a drug abuser.
\end{abstract}

Keywords: alcohol, autonomic nervous system, crack/cocaine, near infrared spectroscopy, prefrontal cortex

\section{Introduction}

Chronic alcohol ingestion is a serious problem around the globe and is related to 3.3 million deaths per year ("Alcohol Facts and Statistics | National Institute on Alcohol Abuse and Alcoholism (NIAAA)," n.d.). It may lead to psychosocial issues, such as anxiety, depression, antisocial and suicidal behaviors (Blanco-Gandía et al., 2015). Also, it is shown to affect cardiovascular function (Whitman et al., 2017), to deteriorate and decrease oxygenation in the prefrontal cortex (PFC) (Schecklmann et al., 2007), and to reduce brain volume in some prosencephalic regions (Wang et al., 2016). Despite alcohol beverages are legal to consume, they might trigger the use of illegal chemical substances such as cocaine and crack (Griffin et al., 2017; Welte \& Barnes, 1985). Crack/cocaine is shown to cause endothelial dysfunction, accelerated atherosclerosis, alterations in blood flow (Bachi et al., 2017) and elevation of adrenergic activity (Fischbach, 2017). Moreover, anxiety, depression and decreased quality of life are also reported in crack/cocaine users (Paiva et al., 2017). In the brain, studies have demonstrated lower dopamine levels (Areal, Herlinger, Pelição, Martins-Silva, \& Pires, 2017) associated with a lower concentration of dopamine D2 receptors in the PFC and a lower firing rate of dopaminergic neurons that project their axons from the ventral tegmental area to areas of the reward system (i.e. PFC, nucleus accumbens, striatum, hippocampus, and amygdala) (Volkow, Fowler, Wang, Baler, \& Telang, 2009).

Both, alcohol and crack/cocaine, have been related to PFC dysfunctions (Goldstein \& Volkow, 2011; Weller et al., 2011) and this damage can persist for months even when the individuals stop using it (Moeller \& Paulus, 2018; Ruan et al., 2017). Importantly, the PFC plays a critical role in impulsivity control and regulation of emotional states, thus, when damaged, it might lead to poor decision-making and further substance use seeking behavior (Goldstein \& Volkow, 2011). Additionally, PFC contributes to autonomic nervous system (ANS) regulation 
(Thayer \& Lane, 2009). Both drugs can cause the withdrawal of parasympathetic tone into the sinoatrial node and increase sympathetic activation (Reed, Porges, \& Newlin, 1999), thereby increasing the risk of vascular events in the brain and the heart (Melillo et al., 2015; Süfke, Fiedler, Djonlagiç, \& Kibbel, 2009). Pharmacotherapy has been pointed as the main treatment option, however there are no consistency in this practice (Thompson, Owens, Pushpakom, Faizal, \& Pirmohamed, 2015). It might lead to gastrointestinal problems, drowsiness, peripheral neuropathy and addiction (Connor, Haber, \& Hall, 2016). Thus, alternative strategies in the rehabilitation and treatment of this disorder are necessary.

Regularly-performed aerobic exercise has been considered an effective tool for treatment and prevention of several pathologies (Salus, 2009). Previous studies have shown the wide range of benefits that exercise promotes on frontal regions of the brain (Hillman, Erickson, \& Kramer, 2008; Tempest \& Parfitt, 2016), cognition, ANS control and psychosocial aspects (Bahrke \& Morgan, 1978; Flausino, Da Silva Prado, de Queiroz, Tufik, \& de Mello, 2012). Studies have also suggested aerobic exercise as a possible complementary tool to treat substance use disorder such as alcohol and cocaine (Cabral et al., 2017; Grandjean da Costa et al., 2017; Manthou et al., 2016; Roessler, 2010). Despite the wide range of benefits, aerobic exercise is usually performed at low and moderate intensity during long training sessions ( $>30 \mathrm{~min}$ ), becoming monotonous and only having the effects experienced after several weeks. Thus, in order to optimize these benefits, high intensity interval training (HIIT), consisting of high-intensity exercise bouts interspersed with intervals of passive or active recovery, has recently emerged in the scientific literature as an interesting strategy for improving cardiorespiratory fitness (Allen et al., 2017), health outcomes and cognitive functions (Costigan, Eather, Plotnikoff, Hillman, \& Lubans, 2016) compared to continuous moderate exercise. Although this exercise modality has been applied in different clinical populations, little is known about its chronic effects among drug users.

Furthermore, neurobiological approaches are important for understanding the possible benefits of high intensity exercise training in a population with an impaired PFC, such as alcohol and crack/cocaine users. In the present case study, we have monitored the effects of a high intensity exercise training protocol on the brain of a male alcohol and crack/cocaine user. We have assessed PFC oxygenation and cerebral electrophysiological responses, as well as cognitive performance, ANS control and psychosocial parameters. We hypothesized that high intensity exercise training would improve PFC functioning and increase parasympathetic tone, cognitive performance and psychosocial aspects. These improvements might help the subject to develop better cognitive control over drug cravings and prevent relapse during treatment.

\section{Methods}

\subsection{Subject}

The subject was a 32-year-old male (weight: $68.1 \mathrm{~kg}$; height: $1.84 \mathrm{~m}$ ) who has been a crack/cocaine and alcohol abuser for 20 years. He was diagnosed having severe substance use disorder according to the Diagnosis Statistical Manual of Mental Disorders, fifth edition (DSM-V), and was in a rehabilitation house implementing religious approaches for the treatment of chemical dependence, which did not involve medication use. The subject was in abstinence for approximately one year when we proposed the study. All the procedures were explained to the patient and a clear and informed consent form was signed before beginning the study. The present study is in accordance with the Helsinki Declaration and was approved by the local ethics committee (number: 51836215.3.0000.5537).

\subsection{Experimental Design}

The volunteer underwent an initial screening in order to check his physical condition to exercise. After, blood pressure measures and questionnaires for physical activity readiness, as well as the application of the Mini Mental state exam (volunteer should score more than 24) were initially assessed to exclude cardiovascular or mental issues. In addition, we recorded patient's resting heart rate variability (HRV) and we conducted psychosocial questionnaires, such as sleep quality, negative emotional states and quality of life responses. Thereafter, the subject performed the Stroop test while electroencephalogram (EEG) measured brain activity. Next, an incremental exercise test on a treadmill was done. During this test, the subject had his PFC oxygenation measured. All tests were done before the exercise program (pre) and after 30 days (12 sessions). All the tests were performed in a single day. Figure 1 demonstrates the experimental design and the high intensity exercise protocol done with the patient. 


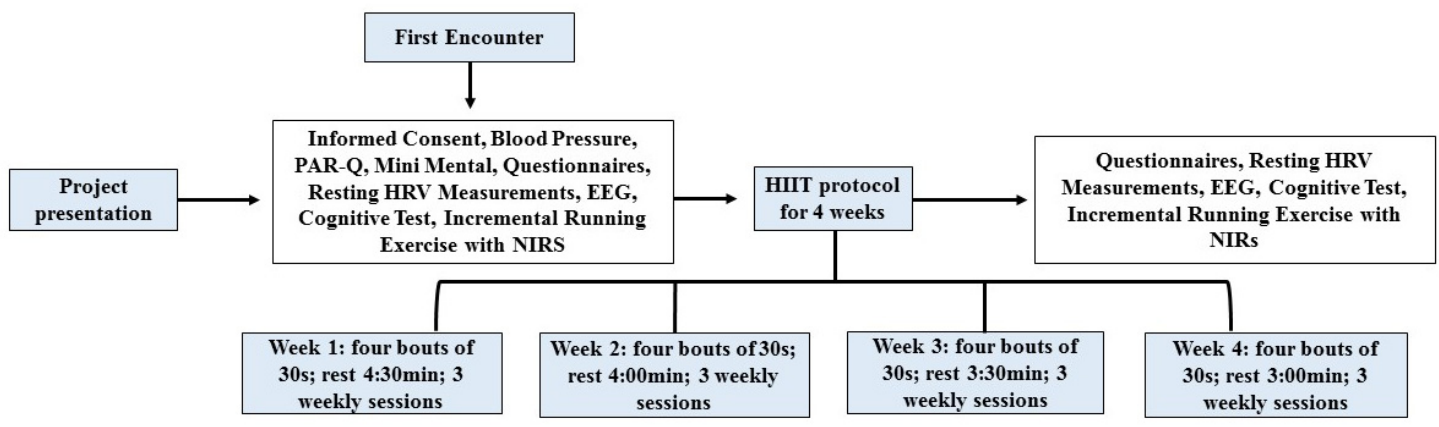

Figure 1. Experimental design done before and after the high intensity exercise protocol with an alcohol and crack/cocaine user under treatment

Abbreviations: HRV: Heart Rate Variability; EEG: Electroencephalogram; NIRS: Near-Infrared spectroscopy; HIIT: High intensity interval training.

\subsection{High Intensity Exercise Protocol}

The protocol consisted of 3 sessions per week for four weeks. Four 30-second bouts were performed in every session from the beginning to the end of the program. The Borg scale of perceived exertion (from 6 to 20) was taught to the volunteer. The subject was instructed to keep the perceived intensity higher than 15 on Borg Scale to characterize a high intensity exercise (Ciolac et al., 2015). The patient rested for four and a half minutes after each bout. Rest time was reduced by 30 s to increase workload in each following week.

\subsection{Incremental Exercise Test}

Prior to this test, the patient received standard instructions on how to report their perceived exertion using the Borg scale (6 to 20). After familiarization with the scale, he was instructed to run in a treadmill until reaching 20 (exhaustion) on the Borg scale during the exercise test, which means complete exhaustion. Thereafter, a warm-up was performed for 4 minutes at $4 \mathrm{~km} / \mathrm{h}$ followed by the test. The test began with the first four minutes at a speed of $6 \mathrm{~km} / \mathrm{h}$ and $5 \%$ incline, followed by an increased $0.5 \mathrm{~km} / \mathrm{h}$ per minute until exhaustion. The evaluator showed the Borg scale every minute, and the patient had to point to the number that represented how hard the exercise was at that moment. A red button in front of the patient was available to stop the test for any intercurrence.

\subsection{Electroencephalography (EEG)}

The patient's electroencephalographic activity was recorded while the Stroop test (see next subsection for more details about the Stroop test) was performed. The EEG cap size was adapted to the participant's head size (Easycap, Herrsching, Germany) using FPz and $\mathrm{Cz}$ as references, obeying the distribution of the 10-20 international system, with 64 active $\mathrm{Ag}-\mathrm{AgCl}$ electrodes (Act-cap, Herrsching, Germany). To set the impedance, $\mathrm{FCz}$ was used as reference and $\mathrm{AFz}$ as ground electrodes. The maximum impedance accepted was $15 \mathrm{k} \Omega$ and the sample rate was $1000 \mathrm{~Hz}$ during acquisition. EEG data was recorded using the Brain Vision Recorder (Brain Products, Muchen, Germany) and processed offline using EEGLAB v.14.0.0 toolbox (Delorme \& Makeig, 2004). In offline signal processing, a band pass filter was amplified at $1-55 \mathrm{~Hz}$, and the data was then re-referenced for average-reference. Independent Components Analysis (ICA) was used to determine artifact rejection (i.e. eye blink and muscle movements) and plot dipoles of activation areas. Frontal lobe (area of interest) components were analyzed before and after the exercise protocol conditions through topographic analysis.

\subsection{Inhibitory Cognitive Control Test (Stroop Test)}

A computer version of the Go/Nogo Stroop matching task was used to measure cognitive performance, where a color bar needs to be associated with the word containing or not containing the interference color (incongruent and congruent trials, respectively) (Caldas, Machado-Pinheiro, Souza, Motta-Ribeiro, \& David, 2012). Note that incongruent trials have interference components, which forces the subject to inhibit a parallel stimulus to respond correctly. A GO response is when the color bar corresponds to the word meaning, and a NOGO response is when this relationship does not exist. The test contained 60 congruent, 60 incongruent and 45 neutral stimulus randomized trials and divided into three blocks. In the GO answers, the participant should press button number "1" on the keyboard, and for the NOGO responses he should not press any button. The patient was instructed to 
respond correctly and as fast as possible. The performance was measured by the number of errors on incongruent trials and by the Stroop effect reaction time. The Stroop effect was calculated by mean reaction time of incongruent trials minus the mean reaction time of congruent trials. Lastly, the software used for this testing was E-prime version2.0 (Psychology Software Tools, Inc, Sharpsburg, PA)

\subsection{Near-Infrared Spectroscopy (NIRS)}

Brain oxygenation was measured by functional Near-Infrared Spectroscopy (NIRS) (Imagent, ISS, Champaign, IL, USA). The NIRS allows quantification of oxyhemoglobin $\left(\mathrm{O}_{2} \mathrm{Hb}\right)$, deoxyhemoglobin $(\mathrm{HbH})$ and total hemoglobin $(\mathrm{HbT})$ according to absorbance at specific wavelengths (Ekkekakis, 2009). We used $\mathrm{O}_{2} \mathrm{Hb}$ since it is the best indicator of cerebral blood flow changes, according to Hoshi and colleagues (Hoshi, Kobayashi, \& Tamura, 2001). In addition, we used the difference between $\mathrm{O}_{2} \mathrm{Hb}$ and $\mathrm{HbH}$ (Hbdiff) as a cerebral activation marker (Hoshi et al., 2001). The NIRS contains two optodes, each containing one detector and four light source pairs. Each pair consisted of two wavelengths $(690$ and $830 \mathrm{~nm})$ and they were positioned at different distances from the detector $(1.5 ; 2.5 ; 3.5$ and $4.5 \mathrm{~cm})$. The optodes were positioned over the patient's Fp1, Fpz and Fp2 regions, according to the international EEG 10-20 distribution system, and were tied with a black bandage to avoid light entrance and optode movement noises. The equipment was calibrated before each evaluation using a calibration phantom and a check phantom that have specific absorption and dispersion coefficients. Data were analyzed in Homer2 (software that runs inside MatLab), provided by the National Institute of Health (P41-RR14075, R01-EB006385). Six minutes rest was exported and normalized in order to explicit the changes magnitude $(\Delta)$. Thereby, we were to analyze the oxygenation changes in the PFC during exercise. This protocol was conducted before and after the exercise training.

\subsection{Heart Rate Variability (HRV)}

HRV measurement was conducted in order to analyze the functionality of the ANS before and after the exercise protocol. The patient was instructed to relax in the dorsal decubitus position. R-R interval recording was conducted for ten minutes and was only started when the respiratory rate was below 20 breaths/minute. A RSC $800 \mathrm{CX}$ heart rate monitor (Polar ${ }^{\circledR}$, Finland) was used with a recording interval at every five seconds. The most stable five-minutes was used to record the parasympathetic indices (SDNN, RMSSD, pNN50 and high frequency power) (Berntson et al., 1997; Malik, 1996). All HRV analysis were performed in the Kubius HRV software, which runs inside the MatLab.

\subsection{Psychosocial Questionnaires}

Three questionnaires were used to evaluate psychosocial status in the initial interview and after the exercise protocol. First, the psychometric scale DASS-21 (Depression, Anxiety and Stress Scale) (Lovibond \& Lovibond, 1995) was used and adapted to local language (Ribeiro, Honrado, \& Leal, 2004). This scale has 21 questions divided into three domains which assess the depression, anxiety and stress levels and have a score ranging from $0 \%$ to $100 \%$ in each of the three domains.

Second, quality of life was assessed using a reduced WHOQOL-100 questionnaire validated for the local population (WHOQOL-brief) (Fleck et al., 2000). This questionnaire has 26 questions. The first one refers to general quality of life and the second corresponds to satisfaction with their own health. The remaining 24 are divided into physical, social, psychological and environmental domains. The score ranges from 0 (worst score) to 100 (best score) for each domain of the questionnaire.

Lastly, the Pittsburgh Sleep Quality questionnaire was used for sleep evaluation. It was validated for the local population as the Pittsburgh Sleep Quality Index (PSQI-BR) and has been used in populations with psychiatric and sleep disorders (Bertolazi et al., 2011). The score was divided into "good sleep" (0 to 4 points), "poor sleep" (5 to 10 points) and "sleep disorders" (> 10 points).

\section{Results}

\subsection{Electroencephalography (EEG)}

Figure 2 shows the topographic responses of EEG frontal components analysis during the Stroop test. Greater activity (U.A) of the frontal area in post-training condition (red means more activation) is observed compared to pre (blue means less activation). On the Stroop test, the patient decreased the number of errors on the incongruent trials by $57.14 \%$ (from 7 to 3) (Panel B), decreased the reaction time of incongruent triais by $6.11 \%$ (from $1010.84 \mathrm{~ms}$ to $949.02 \mathrm{~ms}$ ) (Panel C) and decreased the Stroop effect reaction time (see the methods section for more detail) by $327 \%$ (from $176 \mathrm{~ms}$ to $40.6 \mathrm{~ms}$ ) (Panel D). 


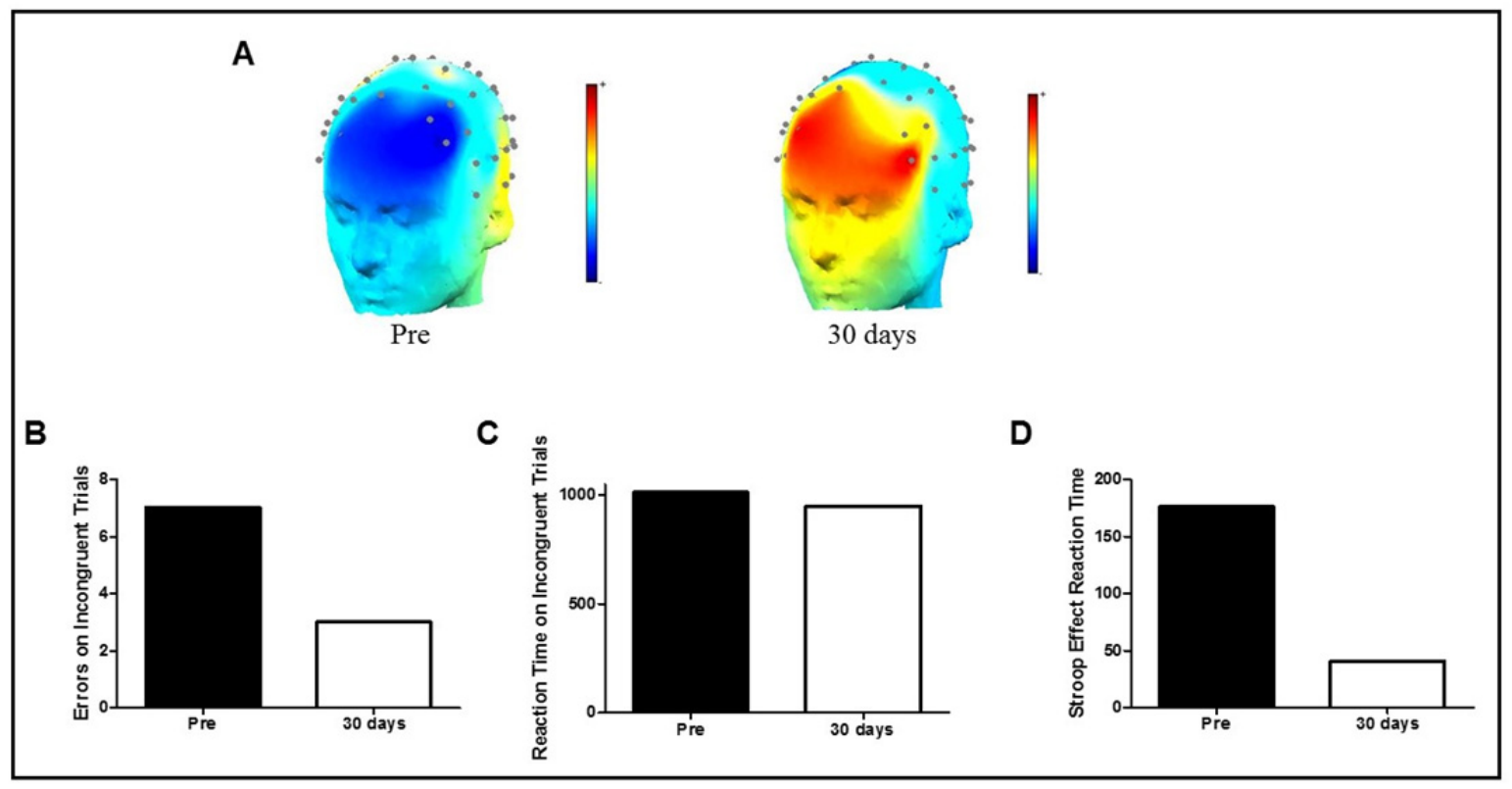

Figure 2. Brain topographic analysis of the drug abuser and his performance on the Stroop test

Panel A: Topographic analysis before and after the training. Panel B: Errors during the incongruent trials of the Stroop test. Panel C: Incongruent trials reaction time on the Stroop test. Panel D: Reaction time during the Stroop effect (incongruent trials - congruent trials).

\subsection{Near Infrared Spectroscopy (NIRS)}

Figure 3 illustrates the PFC hemodynamics during the incremental test evaluated before and 30 days after the high intensity exercise program. The $\mathrm{O}_{2} \mathrm{Hb}$ levels at $10 \%$ of the exercise test increased $228.2 \%$; at $50 \%$ increased $305.4 \%$; and at $100 \%$ increased $359.4 \%$. The HbDiff at the $10 \%$ of the treadmill time decreased $12.8 \%$; at $50 \%$ increased $124.9 \%$; and at $100 \%$ increased $85 \%$. All parameters are comparing pre and 30 days after the high intensity exercise program.

\section{A}

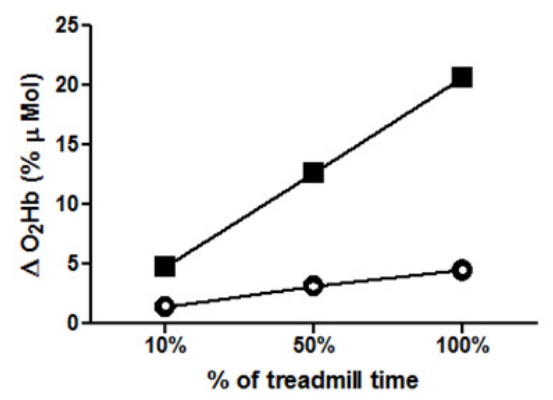

B

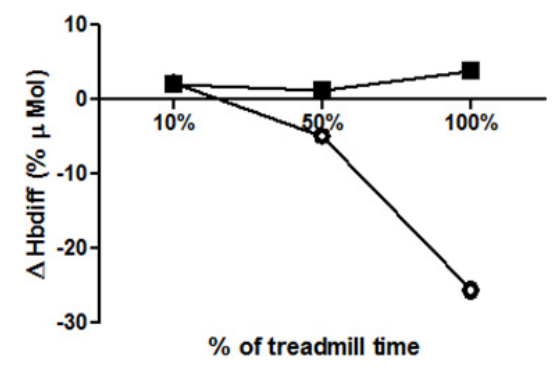

Figure 3. Prefrontal cortex hemodynamics of the drug abuser under rehabilitation while performing incremental exercise test pre and 30 days after the high intensity exercise program

Panel A: Oxyhemoglobin $\left(\mathrm{O}_{2} \mathrm{Hb}\right)$. Panel B: The difference between $\mathrm{O}_{2} \mathrm{Hb}$ and $\mathrm{HbH}$ (HbDiff).

\subsection{Heart Rate Variability}

Figure 4 demonstrates resting HRV indices pre and 30 days after the exercise protocol program. On the time domain indices, RMSSD went from $39.1 \mathrm{~ms}$ to $69.4 \mathrm{~ms}$ (77.4\%), SDNN went from $46.9 \mathrm{~ms}$ to $86 \mathrm{~ms}(83.3 \%)$ and Pnn50 went from 66 to 104 (57.5\%). Regarding the frequency domain indices, high frequency power (HF) went 
from $296 \mathrm{~ms}^{2}$ to $1164 \mathrm{~ms}^{2}(293.2 \%)$. All indices are comparing pre and 30 days after exercise protocol program.

A

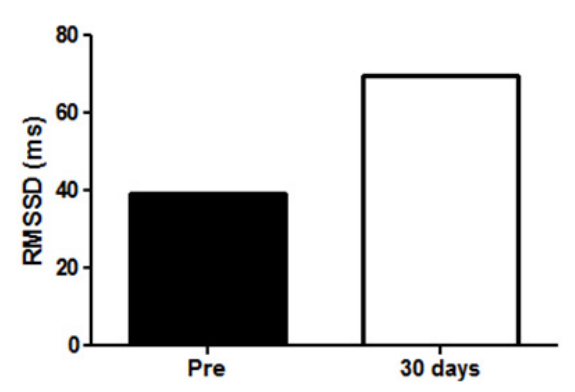

C

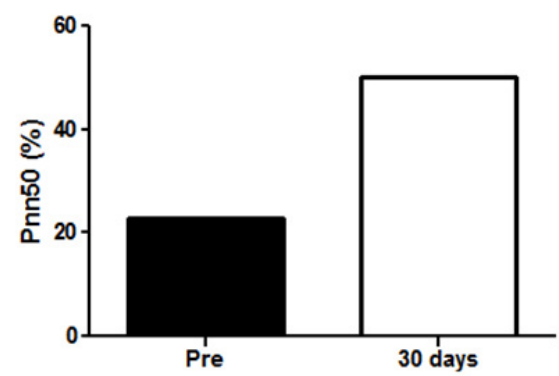

B

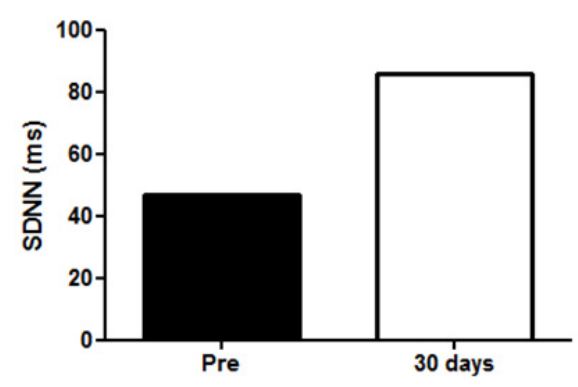

D

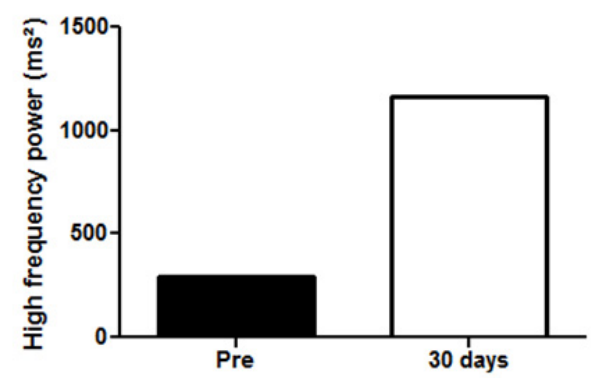

Figure 4. Resting heart rate variability indices before and after 30 days of the high intensity exercise program performed by a drug user under rehabilitation

Panel A: Root mean square of successive differences of R-R intervals (RMSSD); Panel B: Standard deviation of normal to normal R-R intervals (SDNN); Panel C: Percentage of the numbers of interval differences of successive RR intervals greater than 50 ms (Pnn50 \%); Panel D: High frequency power.

\subsection{Psychosocial Questionnaires}

Table 1 shows the enhancements in the psychosocial aspects before and after the high intensity exercise protocol. The subject decreased his score on the sleep quality questionnaire by $23 \%$, which means he went from "sleep disorder" to "bad sleep". On the DASS-21, the volunteer decreased his anxiety levels by $52.6 \%$. Lastly, the psychological domain on the WHOOQOL-brief increased by $5.3 \%$ and the social domain by $13.7 \%$.

Table 1. Questionnaire scores and percentage change of sleep quality, social and psuchological domains and level of anxiety of a drug user under treatment

\begin{tabular}{llll}
\hline Questionnaires & Pre & $\mathbf{3 0}$ days & \% \\
\hline Sleep quality & 13 & 10 & -23 \\
DASS-21 & & & \\
Anxiety & 19 & 9 & -52.6 \\
WHOOQOL-brief & & & \\
Psychological domain & 75 & 79 & 5.3 \\
Social domain & 58 & 66 & 13.7 \\
\hline
\end{tabular}

DASS-21: Depression, Anxiety and Stress-21;

WHOOQOL-brief: World Health Organization Quality of Life - brief. 


\subsection{Treadmill Incremental Test}

Figure 5 demonstrates the incremental exercise test variables before and after the exercise protocol. The test duration increased from 16 minutes to 18 minutes (12.5\%) comparing pre and 30 days after the program. Maximum speed went from $12 \mathrm{~km} / \mathrm{h}$ to $13 \mathrm{~km} / \mathrm{h}(8.3 \%)$. Mean heart rate during incremental test decreased from 176 bpm to $156 \mathrm{bpm}(-11.3 \%)$.

A

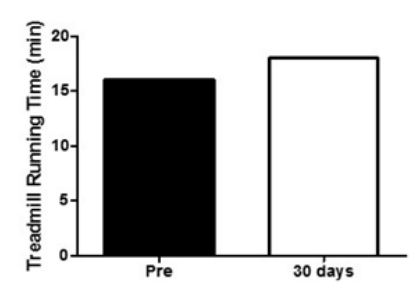

B

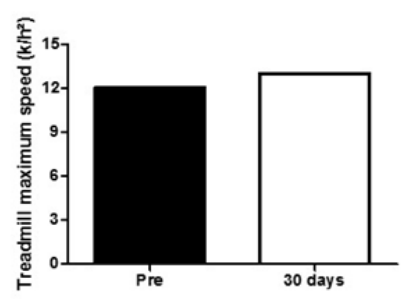

C

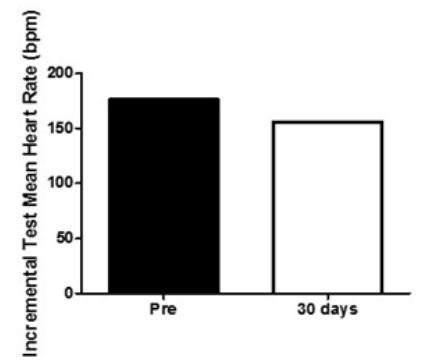

Figure 5. Effects of the high intensity exercise program on the incremental exercise test performance of a drug user under treatment

Panel A: Treadmill running time before and 30 days after the beginning of the high intensity exercise program. Panel B: Treadmill maximum speed achieved before and 30 days after the begging of the high intensity exercise program. Panel C: Mean heart rate during the incremental test before and 30 days after the begging of the high intensity exercise program.

\section{Discussion}

To the best of our knowledge, this is a pioneering work that investigates the effects of high intensity exercise training on the brain of an alcohol and crack/cocaine user. The main findings of this case study indicate that a 30-day high intensity exercise program, performed by a patient under treatment, promoted improvements in PFC functioning, as well as in autonomic cardiac control and psychosocial parameters.

It is well known that drug addicted individuals have impaired PFC functions (Verdejo-Garcia et al., 2015; Wang et al., 2016). Studies have shown that the PFC plays an important role in the addictive behavior (Koob \& Volkow, 2009), and damage to this area seems to be critical in drug-seeking behaviors (Volkow, Koob, \& McLellan, 2016). This specific region is involved in several cognitive functions including inhibitory control, attention, working memory and decision-making (Diamond, 2013). Thus, strategies that increase the functionality of this area might be crucial for these individuals. Interestingly, high intensity exercise has shown to promote positive effects on the brain, increasing its metabolism and cognitive functions in a healthy sample (Coetsee \& Terblanche, 2017; Voelcker-Rehage \& Niemann, 2013). Our data show similar benefits on the patient's brain. The individual acutely and chronically increased his PFC oxygenation during exercise (see Figure 3), and these metabolism changes are in conformity with previous findings that presented greater brain oxygenation during incremental exercise (Bediz et al., 2016; Chang et al., 2013; Tempest \& Parfitt, 2016). Improvements in the oxygen delivered to the PFC can possibly be related to increased number of angiogenic factors due to the exercise program, thus increasing the number of capillaries in the region (Ding et al., 2006; Swain et al., 2003).

Furthermore, the topographic analysis was used to investigate changes in the volunteer's brain activity (Liskow, 2002). In this analysis, the subject showed enhanced PFC activity during the Stroop test, as well as improved performance on the cognitive test (see Figure 2). Previous studies have shown that patients with substance use disorder usually exhibit decreased inhibitory response on the Stroop test (Hillman \& Drobes, 2012), and that an acute session of aerobic exercise improves the performance on inhibitory control associated with greater activity on electrophysiological records of the PFC (Wang, Zhou, Zhao, Wu, \& Chang, 2016). Interestingly, exercise has been related to increased levels of brain-derived neurotrophic factor, which plays an important role on several molecular changes that might enhance cognitive functions (Monnier et al., 2017). In addition, exercise has also been shown to improve neurogenesis (van Praag, 2008). Taken together, these PFC and cognitive improvements are extremely important to this individual, because it might lead to greater impulsivity control and abstinence maintenance, thereby decreasing the negative effects on cognitive functions induced by chronic drug intake. 
Furthermore, attention bias is common in patients with addictive disorder (Zhang et al., 2018) and the left dorsolateral PFC plays a key role in attention bias (Heeren, Baeken, Vanderhasselt, Philippot, \& de Raedt, 2015). In addition, the hypoactivation of the PFC is associated with depression (Ho, Zhang, \& Ho, 2016), which is a common comorbidity of addiction (Puri et al 2014). High intensity exercise may activate PFC and reduce the severity of depression as well as attention bias in people who misuse alcohol and cocaine.

It is well known that crack/cocaine and alcohol chronic use might have negative effects on ANS balance (de Zambotti et al., 2014; Fischbach, 2017), leading to increased sympathetic activity, which can increase the risk of cardiovascular and cerebrovascular events (Melillo et al., 2015). This is because enhanced adrenergic activity is related to greater oxygen requirement by the heart, leading to arrhythmias and vascular oxidative stress (Caetano \& Delgado Alves, 2015). Arguably, the literature is clear regarding the evidence of physical activity benefits in ANS balance and improvements in cardiovascular health (Fu \& Levine, 2013). Curiously, high intensity exercise has been related to promote superior ANS benefits than usual moderate aerobic exercise, since it has been shown to have greater angiotensin II reduction, with consequent disinhibition of cardiac vagal function and increased nitric oxide availability, which leads to decreased sympathetic tone and enhance baroreflex sensitivity (Bhati, Bansal, \& Moiz, 2017). In the present study, we measured ANS control through HRV before and after the training program, and we found several improvements on HRV vagal indices (see Figure 4). These improvements reflect better ANS control with higher parasympathetic activity, leading to greater HRV. Enhanced HRV is shown to be associated with better cognitive performance and better decision making. This might be explained by the fact that the PFC plays a key role in ANS regulation through inhibitory function over the amygdala and its control over sympathetic neurons on the brainstem (Thayer \& Lane, 2009). Conversely, lower HRV is related to increased susceptibility to stress and anxiety (Thayer, Friedman, \& Borkovec, 1996), which leads the individual to look for a way out of these emotional states through substance abuses, for example (Volkow et al., 2016). Furthermore, higher HRV is also related to better response to negative stimuli imposed by the environment (Porges, 1995). Therefore, enhanced HRV seems to be important to this patient because of its association with better decision-making and control over impulsivity, possibly leading to a better control over the substance craving and helping his rehabilitation.

Psychological symptoms such as anxiety, decreased sleep quality, and poor quality of life are common in drug abuse, and these problems might compromise the treatment outcome (Chakravorty, Chaudhary, \& Brower, 2016; de Zambotti et al., 2014). However, exercise seems to act on several mechanisms, promoting a decrease in these symptoms in drug abusers (Linke \& Ussher, 2015; Ussher, Sampuran, Doshi, West, \& Drummond, 2004; Williams et al., 2011). In the present research, we have showed that the high intensity exercise program was capable to improve sleep quality, quality of life (social domain and psychological domain) and reduce anxiety levels of the subject (see Table 1). It is known that alcoholic subjects may develop sleep quality problems and this is associated with depression (Garfield, Llewellyn, \& Kumari, 2016) and negative impacts on physical and mental health (da Silva et al., 2016). Importantly, studies have reported improved sleep quality for those who regularly practice exercise (Driver \& Taylor, 2000). Additionally, higher anxiety levels have been shown to be present in chronic drug users (Regier, Rae, Narrow, Kaelber, \& Schatzberg, 1998) and regular exercise has also been demonstrated to be an effective tool to reduce this symptom. This is because exercise reduces muscle tension and promotes sympathetic activity, which then induces higher parasympathetic activity after the exercise and might distract people from daily stressful events (Callaghan, 2004). Certainly, these improvements influenced patient's quality of life increasing, especially in the psychological domain (see Table 1). Moreover, the social domain was also improved and this might be due to the development of healthy relationships with the team that was applying the training three times a week. Furthermore, the subject performed the high intensity exercise protocol while other individuals with drug problems were running or walking around the same place. Thus, social interaction was encouraged after training. We believe that the enhancements in psychosocial parameters might facilitate improvements in social skills and thus reducing negative emotional states, contributing to the treatment and rehabilitation of the volunteer.

Lastly, it is worth mentioning that the patient improved his cardiorespiratory aspects, as shown in Figure 5, which means the training protocol has enhanced his cardiorespiratory fitness. A possible mechanism in which high intensity exercise enhances the cardiovascular system is by increasing maximal stroke volume, cardiac output and skeletal muscle mitochondrial oxidative capacity (Daussin et al., 2008). As evidenced in the literature, drug users usually present higher risk of cardiovascular problems such as atrial fibrillation and congestive heart failure (Whitman et al., 2017). Hence, increasing cardiovascular functioning through high intensity exercise training is a very important aspect to be considered.

We assume the limitations of a case study where conversions to general populations with substance use disorders cannot be made. However, long-term studies verifying the effects of exercise on individuals with drug addiction 
are lacking in the literature, especially with neurobiological, cognitive, cardiovascular and psychosocial markers as our case study shows. Moreover, we also acknowledge that the improvements shown here are not exclusive effects of exercise, especially on the psychosocial aspects, which might be the effects of other approaches in the rehabilitation treatment. However, it is very unlikely that the improvements in cardiovascular, brain hemodynamics and brain activity are related to approaches other than exercise, as they are physiological adaptations to exercise.

\section{Conclusion}

In summary, it is concluded that a 30-day high intensity exercise program improved PFC functionality and cognitive performance. Moreover, autonomic nervous control was also enhanced, as well as psychosocial factors. We believe that these benefits caused by a high intensity exercise program may promote higher control of the drug seeking behavior and decrease chances of relapse. Therefore, it is possible to speculate that the high intensity exercise could be a usefull tool to help the treatment of drug users.

\section{Competing Interests Statement}

The authors report no conflict of interest.

\section{References}

Alcohol Facts and Statistics | National Institute on Alcohol Abuse and Alcoholism (NIAAA). (n.d.).

Allen, N. G., Higham, S. M., Mendham, A. E., Kastelein, T. E., Larsen, P. S., \& Duffield, R. (2017). The effect of high-intensity aerobic interval training on markers of systemic inflammation in sedentary populations. European Journal of Applied Physiology. https://doi.org/10.1007/s00421-017-3613-1

Areal, L. B., Herlinger, A. L., Pelição, F. S., Martins-Silva, C., \& Pires, R. G. W. (2017). Crack cocaine inhalation induces schizophrenia-like symptoms and molecular alterations in mice prefrontal cortex. Journal of Psychiatric Research, 91, 57-63. https://doi.org/10.1016/j.jpsychires.2017.03.005

Bachi, K., Mani, V., Jeyachandran, D., Fayad, Z. A., Goldstein, R. Z., \& Alia-Klein, N. (2017). Vascular disease in cocaine addiction. Atherosclerosis. https://doi.org/10.1016/j.atherosclerosis.2017.03.019

Bahrke, M. S., \& Morgan, W. P. (1978). Anxiety reduction following exercise and meditation. Cognitive Therapy and Research, 2(4), 323-333. https://doi.org/10.1007/BF01172650

Bediz, C. S., Oniz, A., Guducu, C., Ural Demirci, E., Ogut, H., Gunay, E., ... Ozgoren, M. (2016). Acute Supramaximal Exercise Increases the Brain Oxygenation in Relation to Cognitive Workload. Frontiers in Human Neuroscience, 10, 174. https://doi.org/10.3389/fnhum.2016.00174

Berntson, G. G., Bigger, J. T., Eckberg, D. L., Grossman, P., Kaufmann, P. G., Malik, M., ... van der Molen, M. W. (1997). Heart rate variability: origins, methods, and interpretive caveats. Psychophysiology, 34(6), 623-648.

Bertolazi, A. N., Fagondes, S. C., Hoff, L. S., Dartora, E. G., Miozzo, I. C. da S., de Barba, M. E. F., \& Barreto, S. S. M. (2011). Validation of the Brazilian Portuguese version of the Pittsburgh Sleep Quality Index. Sleep Medicine, 12(1), 70-75. https://doi.org/10.1016/j.sleep.2010.04.020

Bhati, P., Bansal, V., \& Moiz, J. A. (2017). Comparison of different volumes of high intensity interval training on cardiac autonomic function in sedentary young women. International Journal of Adolescent Medicine and Health. https://doi.org/10.1515/ijamh-2017-0073

Blanco-Gandía, M. C., Mateos-García, A., García-Pardo, M. P., Montagud-Romero, S., Rodríguez-Arias, M., Miñarro, J., \& Aguilar, M. A. (2015). Effect of drugs of abuse on social behaviour: a review of animal models. Behavioural Pharmacology, 26(6), 541-570. https://doi.org/10.1097/FBP.0000000000000162

Cabral, D. A., da Costa, K. G., Okano, A. H., Elsangedy, H. M., Rachetti, V. P., \& Fontes, E. B. (2017). Improving cerebral oxygenation, cognition and autonomic nervous system control of a chronic alcohol abuser through a three-month running program. Addictive Behaviors Reports. https://doi.org/10.1016/j.abrep.2017.08.004

Caetano, J., \& Delgado Alves, J. (2015). Heart rate and cardiovascular protection. European Journal of Internal Medicine, 26(4), 217-222. https://doi.org/10.1016/j.ejim.2015.02.009

Caldas, A. L., Machado-Pinheiro, W., Souza, L. B., Motta-Ribeiro, G. C., \& David, I. A. (2012). The Stroop matching task presents conflict at both the response and nonresponse levels: an event-related potential and $\begin{array}{llll}\text { electromyography } & \text { study. } & \text { Psychophysiology, } & 49(9),\end{array}$ https://doi.org/10.1111/j.1469-8986.2012.01407.x

Callaghan, P. (2004). Exercise: a neglected intervention in mental health care? Journal of Psychiatric and Mental 
Health Nursing, 11(4), 476-483. https://doi.org/10.1111/j.1365-2850.2004.00751.x

Chakravorty, S., Chaudhary, N. S., \& Brower, K. J. (2016). Alcohol Dependence and Its Relationship With Insomnia and Other Sleep Disorders. Alcoholism, Clinical and Experimental Research, 40(11), 2271-2282. https://doi.org/10.1111/acer.13217

Chang, H., Kim, K., Jung, Y.-J., Ahn, N.-R., So, W.-Y., \& Kato, M. (2013). Effects of blood flow to the prefrontal cortex on high-intensity exercise combined with high-decibel music. Journal of Exercise Nutrition \& Biochemistry, 17(4), 115-122. https://doi.org/10.5717/jenb.2013.17.4.115

Ciolac, E., Mantuani, S., Neiva, C., Verardi, C., Pessôa-Filho, D., \& Pimenta, L. (2015). Rating of perceived exertion as a tool for prescribing and self regulating interval training: a pilot study. Biology of Sport, 32(2), 103-108. https://doi.org/10.5604/20831862.1134312

Coetsee, C., \& Terblanche, E. (2017). Cerebral oxygenation during cortical activation: the differential influence of three exercise training modalities. A randomized controlled trial. European Journal of Applied Physiology, 117(8), 1617-1627. https://doi.org/10.1007/s00421-017-3651-8

Connor, J. P., Haber, P. S., \& Hall, W. D. (2016). Alcohol use disorders. Lancet (London, England), 387(10022), 988-998. https://doi.org/10.1016/S0140-6736(15)00122-1

Costigan, S. A., Eather, N., Plotnikoff, R. C., Hillman, C. H., \& Lubans, D. R. (2016). High-Intensity Interval Training for Cognitive and Mental Health in Adolescents. Medicine and Science in Sports and Exercise, 48(10), 1985-1993. https://doi.org/10.1249/MSS.0000000000000993

da Silva, A. A., de Mello, R. G. B., Schaan, C. W., Fuchs, F. D., Redline, S., \& Fuchs, S. C. (2016). Sleep duration and mortality in the elderly: a systematic review with meta-analysis. BMJ Open, 6(2), e008119. https://doi.org/10.1136/bmjopen-2015-008119

Daussin, F. N., Zoll, J., Dufour, S. P., Ponsot, E., Lonsdorfer-Wolf, E., Doutreleau, S., ... Richard, R. (2008). Effect of interval versus continuous training on cardiorespiratory and mitochondrial functions: relationship to aerobic performance improvements in sedentary subjects. American Journal of Physiology. Regulatory, Integrative and Comparative Physiology, 295(1), R264-272. https://doi.org/10.1152/ajpregu.00875.2007

de Zambotti, M., Baker, F. C., Sugarbaker, D. S., Nicholas, C. L., Trinder, J., \& Colrain, I. M. (2014). Poor autonomic nervous system functioning during sleep in recently detoxified alcohol-dependent men and women. Alcoholism: Clinical and Experimental Research, 38(5), 1373-1380. https://doi.org/10.1111/acer.12384

Delorme, A., \& Makeig, S. (2004). EEGLAB: an open source toolbox for analysis of single-trial EEG dynamics including independent component analysis. Journal of Neuroscience Methods, 134(1), 9-21. https://doi.org/10.1016/j.jneumeth.2003.10.009

Diamond, A. (2013). Executive functions. Annual Review of Psychology, 64, 135-168. https://doi.org/10.1146/annurev-psych-113011-143750

Ding, Y.-H., Li, J., Zhou, Y., Rafols, J. A., Clark, J. C., \& Ding, Y. (2006). Cerebral Angiogenesis and Expression of Angiogenic Factors in Aging Rats after Exercise. Current Neurovascular Research, 3(1), 15-23.

Driver, H. S., \& Taylor, S. R. (2000). Exercise and sleep. Sleep Medicine Reviews, 4(4), 387-402. https://doi.org/10.1053/smrv.2000.0110

Ekkekakis, P. (2009). Illuminating the black box: investigating prefrontal cortical hemodynamics during exercise with near-infrared spectroscopy. Journal of Sport \& Exercise Psychology, 31(4), 505-553.

Fischbach, P. (2017). The role of illicit drug use in sudden death in the young. Cardiology in the Young, 27(S1), S75-S79. https://doi.org/10.1017/S1047951116002274

Flausino, N. H., Da Silva Prado, J. M., de Queiroz, S. S., Tufik, S., \& de Mello, M. T. (2012). Physical exercise performed before bedtime improves the sleep pattern of healthy young good sleepers. Psychophysiology, 49(2), 186-192. https://doi.org/10.1111/j.1469-8986.2011.01300.x

Fleck, M. P., Louzada, S., Xavier, M., Chachamovich, E., Vieira, G., Santos, L., \& Pinzon, V. (2000). Application of the Portuguese version of the abbreviated instrument of quality life WHOQOL-bref. Revista de Saúde Pública, 34(2), 178-183. https://doi.org/10.1590/S0034-89102000000200012

Fu, Q., \& Levine, B. D. (2013). Exercise and the autonomic nervous system. Handbook of Clinical Neurology, 117, 147-160. https://doi.org/10.1016/B978-0-444-53491-0.00013-4 
Garfield, V., Llewellyn, C. H., \& Kumari, M. (2016). The relationship between physical activity, sleep duration and depressive symptoms in older adults: The English Longitudinal Study of Ageing (ELSA). Preventive Medicine Reports, 4, 512-516. https://doi.org/10.1016/j.pmedr.2016.09.006

Goldstein, R. Z., \& Volkow, N. D. (2011). Dysfunction of the prefrontal cortex in addiction: neuroimaging findings and clinical implications. Nature Reviews. Neuroscience, 12(11), 652-669. https://doi.org/10.1038/nrn3119

Grandjean da Costa, K., Soares Rachetti, V., Quirino Alves da Silva, W., Aranha Rego Cabral, D., Gomes da Silva Machado, D., Caldas Costa, E., ... Bodnariuc Fontes, E. (2017). Drug abusers have impaired cerebral oxygenation and cognition during exercise. PloS One, 12(11), e0188030. https://doi.org/10.1371/journal.pone.0188030

Griffin, E. A., Melas, P. A., Zhou, R., Li, Y., Mercado, P., Kempadoo, K. A., .. Kandel, D. B. (2017). Prior alcohol use enhances vulnerability to compulsive cocaine self-administration by promoting degradation of HDAC4 and HDAC5. Science Advances, 3(11), e1701682. https://doi.org/10.1126/sciadv.1701682

Heeren, A., Baeken, C., Vanderhasselt, M.-A., Philippot, P., \& de Raedt, R. (2015). Impact of Anodal and Cathodal Transcranial Direct Current Stimulation over the Left Dorsolateral Prefrontal Cortex during Attention Bias

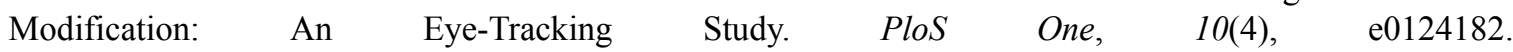
https://doi.org/10.1371/journal.pone.0124182

Hillman, C. H., \& Drobes, D. J. (2012). Physical Activity and Cognitive Control: Implications for Drug Abuse. Child Development Perspectives, 6(4), 367-373. https://doi.org/10.1111/cdep.12000

Hillman, C. H., Erickson, K. I., \& Kramer, A. F. (2008). Be smart, exercise your heart: exercise effects on brain and cognition. Nature Reviews. Neuroscience, 9(1), 58-65. https://doi.org/10.1038/nrn2298

Ho, C. S. H., Zhang, M. W. B., \& Ho, R. C. M. (2016). Optical Topography in Psychiatry: A Chip Off the Old Block or a New Look Beyond the Mind-Brain Frontiers? Frontiers in Psychiatry, 7. https://doi.org/10.3389/fpsyt.2016.00074

Hoshi, Y., Kobayashi, N., \& Tamura, M. (2001). Interpretation of near-infrared spectroscopy signals: a study with a newly developed perfused rat brain model. Journal of Applied Physiology, 90(5), 1657-1662.

Koob, G. F., \& Volkow, N. D. (2009). Neurocircuitry of Addiction. Neuropsychopharmacology, 35(1), 217-238. https://doi.org/10.1038/npp.2009.110

Linke, S. E., \& Ussher, M. (2015). Exercise-based treatments for substance use disorders: evidence, theory, and practicality. The American Journal of Drug and Alcohol Abuse, 41(1), 7-15. https://doi.org/10.3109/00952990.2014.976708

Liskow, B. I. (2002). Brain Imaging in Substance Abuse: Research, Clinical, and Forensic Applications. American Journal of Psychiatry, 159(5), 889-890. https://doi.org/10.1176/appi.ajp.159.5.889

Lovibond, P. F., \& Lovibond, S. H. (1995). The structure of negative emotional states: comparison of the Depression Anxiety Stress Scales (DASS) with the Beck Depression and Anxiety Inventories. Behaviour Research and Therapy, 33(3), 335-343.

Malik, M. (1996). Heart Rate Variability. Annals of Noninvasive Electrocardiology, 1(2), 151-181. https://doi.org/10.1111/j.1542-474X.1996.tb00275.x

Manthou, E., Georgakouli, K., Fatouros, I. G., Gianoulakis, C., Theodorakis, Y., \& Jamurtas, A. Z. (2016). Role of exercise in the treatment of alcohol use disorders. Biomedical Reports, 4(5), 535-545. https://doi.org/10.3892/br.2016.626

Melillo, P., Izzo, R., Orrico, A., Scala, P., Attanasio, M., Mirra, M., ... Pecchia, L. (2015). Automatic Prediction of Cardiovascular and Cerebrovascular Events Using Heart Rate Variability Analysis. PLOS ONE, 10(3), e0118504. https://doi.org/10.1371/journal.pone.0118504

Moeller, S. J., \& Paulus, M. P. (2018). Toward biomarkers of the addicted human brain: Using neuroimaging to predict relapse and sustained abstinence in substance use disorder. Progress in Neuro-Psychopharmacology \& Biological Psychiatry, 80(Pt B), 143-154. https://doi.org/10.1016/j.pnpbp.2017.03.003

Monnier, A., Garnier, P., Quirie, A., Pernet, N., Demougeot, C., Marie, C., \& Prigent-Tessier, A. (2017). Effect of short-term exercise training on brain-derived neurotrophic factor signaling in spontaneously hypertensive rats. Journal of Hypertension, 35(2), 279-290. https://doi.org/10.1097/HJH.0000000000001164

Paiva, C. B., Ferreira, I. B., Bosa, V. L., Narvaez, J. C. de M., Paiva, C. B., Ferreira, I. B., ... Narvaez, J. C. de M. 
(2017). Depression, anxiety, hopelessness and quality of life in users of cocaine/crack in outpatient treatment. Trends in Psychiatry and Psychotherapy, 39(1), 34-42. https://doi.org/10.1590/2237-6089-2015-0065

Porges, S. W. (1995). Orienting in a defensive world: mammalian modifications of our evolutionary heritage. A Polyvagal Theory. Psychophysiology, 32(4), 301-318.

Puri B, Hall A and Ho RC (eds) Revision Notes in Psychiatry. 3rd edn. CRC Press: New York. 2014

Reed, S. F., Porges, S. W., \& Newlin, D. B. (1999). Effect of alcohol on vagal regulation of cardiovascular function: contributions of the polyvagal theory to the psychophysiology of alcohol. Experimental and Clinical Psychopharmacology, 7(4), 484-492.

Regier, D. A., Rae, D. S., Narrow, W. E., Kaelber, C. T., \& Schatzberg, A. F. (1998). Prevalence of anxiety disorders and their comorbidity with mood and addictive disorders. The British Journal of Psychiatry. Supplement, (34), 24-28.

Ribeiro, J. L. P., Honrado, A. A. J. D., \& Leal, I. P. (2004). Contribuição para o estudo da adaptação portuguesa das Escalas de Ansiedade, Depressão e Stress (EADS) de 21 itens de Lovibond e Lovibond. Psicologia, Saúde \& Doenças, 2229-2239.

Roessler, K. K. (2010). Exercise treatment for drug abuse - A Danish pilot study. Scandinavian Journal of Public Health, 38(6), 664-669. https://doi.org/10.1177/1403494810371249

Ruan, X., Zhong, N., Yang, Z., Fan, X., Zhuang, W., Du, J., ... Zhao, M. (2017). Gray matter volume showed dynamic alterations in methamphetamine users at 6 and 12months abstinence: A longitudinal voxel-based morphometry study. Progress in Neuro-Psychopharmacology \& Biological Psychiatry. https://doi.org/10.1016/j.pnpbp.2017.09.004

Salus, R. E. (2009). Exercise is medicine and physicians need to prescribe it! Medicina Dello Sport, 62(4), 517-520. https://doi.org/10.1136/bjsm.2008.054825

Schecklmann, M., Ehlis, A.-C., Plichta, M. M., Boutter, H. K., Metzger, F. G., \& Fallgatter, A. J. (2007). Altered frontal brain oxygenation in detoxified alcohol dependent patients with unaffected verbal fluency performance. Psychiatry Research, 156(2), 129-138. https://doi.org/10.1016/j.pscychresns.2007.01.009

Süfke, S., Fiedler, S., Djonlagiç, H., \& Kibbel, T. (2009). [Continuous analysis of heart rate variability for examination of cardiac autonomic nervous system after alcohol intoxication]. Medizinische Klinik (Munich, Germany: 1983), 104(7), 511-519. https://doi.org/10.1007/s00063-009-1110-y

Swain, R. A., Harris, A. B., Wiener, E. C., Dutka, M. V., Morris, H. D., Theien, B. E., ... Greenough, W. T. (2003). Prolonged exercise induces angiogenesis and increases cerebral blood volume in primary motor cortex of the rat. Neuroscience, 117(4), 1037-1046.

Tempest, G., \& Parfitt, G. (2016). Self-reported tolerance influences prefrontal cortex hemodynamics and affective responses. Cognitive, Affective \& Behavioral Neuroscience, 16(1), 63-71. https://doi.org/10.3758/s13415-015-0374-3

Thayer, J. F., Friedman, B. H., \& Borkovec, T. D. (1996). Autonomic characteristics of generalized anxiety disorder and worry. Biological Psychiatry, 39(4), 255-266. https://doi.org/10.1016/0006-3223(95)00136-0

Thayer, Julian F., \& Lane, R. D. (2009). Claude Bernard and the heart-brain connection: further elaboration of a model of neurovisceral integration. Neuroscience and Biobehavioral Reviews, 33(2), 81-88. https://doi.org/10.1016/j.neubiorev.2008.08.004

Thompson, A., Owens, L., Pushpakom, S. P., Faizal, M., \& Pirmohamed, M. (2015). Pharmacotherapy for alcohol dependence: A stratified approach. Pharmacology \& Therapeutics, 153, 10-24. https://doi.org/10.1016/j.pharmthera.2015.05.010

Ussher, M., Sampuran, A. K., Doshi, R., West, R., \& Drummond, D. C. (2004). Acute effect of a brief bout of exercise on alcohol urges. Addiction, 99(12), 1542-1547. https://doi.org/10.1111/j.1360-0443.2004.00919.x

van Praag, H. (2008). Neurogenesis and exercise: past and future directions. Neuromolecular Medicine, 10(2), 128-140. https://doi.org/10.1007/s12017-008-8028-z

Verdejo-Garcia, A., Clark, L., Verdejo-Román, J., Albein-Urios, N., Martinez-Gonzalez, J. M., Gutierrez, B., \& Soriano-Mas, C. (2015). Neural substrates of cognitive flexibility in cocaine and gambling addictions. The British Journal of Psychiatry: The Journal of Mental Science, 207(2), 158-164. https://doi.org/10.1192/bjp.bp.114.152223 
Voelcker-Rehage, C., \& Niemann, C. (2013). Structural and functional brain changes related to different types of physical activity across the life span. Neuroscience and Biobehavioral Reviews, 37(9), 2268-2295. https://doi.org/10.1016/j.neubiorev.2013.01.028

Volkow, N. D., Fowler, J. S., Wang, G. J., Baler, R., \& Telang, F. (2009). Imaging dopamine's role in drug abuse and addiction. Neuropharmacology, 56 Suppl 1, 3-8. https://doi.org/10.1016/j.neuropharm.2008.05.022

Volkow, Nora D., Koob, G. F., \& McLellan, A. T. (2016). Neurobiologic Advances from the Brain Disease Model of Addiction. The New England Journal of Medicine, 374(4), 363-371. https://doi.org/10.1056/NEJMra1511480

Wang, D., Zhou, C., Zhao, M., Wu, X., \& Chang, Y.-K. (2016). Dose-response relationships between exercise intensity, cravings, and inhibitory control in methamphetamine dependence: An ERPs study. Drug and Alcohol Dependence, 161, 331-339. https://doi.org/10.1016/j.drugalcdep.2016.02.023

Wang, J., Fan, Y., Dong, Y., Ma, M., Ma, Y., Dong, Y., ... Cui, C. (2016). Alterations in Brain Structure and Functional Connectivity in Alcohol Dependent Patients and Possible Association with Impulsivity. PloS One, 11(8), e0161956. https://doi.org/10.1371/journal.pone.0161956

Weller, R. E., Stoeckel, L. E., Milby, J. B., Bolding, M., Twieg, D. B., Knowlton, R. C., ... Ding, Z. (2011). Smaller regional gray matter volume in homeless african american cocaine-dependent men: a preliminary report. The Open Neuroimaging Journal, 5, 57-64. https://doi.org/10.2174/1874440001105010057

Welte, J. W., \& Barnes, G. M. (1985). Alcohol: The gateway to other drug use among secondary-school students. Journal of Youth and Adolescence, 14(6), 487-498. https://doi.org/10.1007/BF02139522

Whitman, I. R., Agarwal, V., Nah, G., Dukes, J. W., Vittinghoff, E., Dewland, T. A., \& Marcus, G. M. (2017). Alcohol Abuse and Cardiac Disease. Journal of the American College of Cardiology, 69(1), 13-24. https://doi.org/10.1016/j.jacc.2016.10.048

Williams, D. M., Dunsiger, S., Whiteley, J. A., Ussher, M. H., Ciccolo, J. T., \& Jennings, E. G. (2011). Acute effects of moderate intensity aerobic exercise on affective withdrawal symptoms and cravings among women smokers. Addictive Behaviors, 36(8), 894-897. https://doi.org/10.1016/j.addbeh.2011.04.001

Zhang, M., Ying, J., Song, G., Ho, R. C., Fung, D. S., \& Smith, H. (2018). Attention Bias in Individuals with Addictive Disorders: Systematic Review Protocol. JMIR Research Protocols, 7(2). https://doi.org/10.2196/resprot.9385

\section{Copyrights}

Copyright for this article is retained by the author(s), with first publication rights granted to the journal.

This is an open-access article distributed under the terms and conditions of the Creative Commons Attribution license (http://creativecommons.org/licenses/by/4.0/). 